\title{
The Evolution of the Primate Prehensile Tail
}

\author{
Emily Xu ${ }^{1}$, Patricia M. Gray ${ }^{1}$ \\ ${ }^{1}$ University of Western Ontario, Department of Biology
}

\begin{abstract}
The evolution of the prehensile tail illustrates the impact habitat can have on structural traits. Prehensile primates are able to support their entire body weight using only their tail, which opens up new feeding opportunities in their arboreal environments. This trait evolved separately in two families of New World monkeys. A transitional behaviour in its proposed evolutionary mechanism is tail-assisted hind limb suspension during locomotion in these dense forests. The evolution of more robust vertebrae, shorter distal vertebrae, a greater number of zygapophyseal joints, as well as larger and more convex articular surfaces, result in a stronger and more flexible tail. Prehensile tails have more expanded muscle attachments that can bear greater loading forces. A naked tactile pad that improves grip is present only in atelids. These differences in bone and muscle morphology make the prehensile tail more sturdy and dexterous, allowing prehensile primates to use their tails for an alternative function.
\end{abstract}

KEYWORDS: Evolution, Primate, Prehensile Tail, Platyrrhine, New World Monkey

This essay is part of a continuing series of student class work completed in a second-year biology course at The University of Western Ontario, introduced here. The essay illustrates one or more principles of evolution on a topic of the student's own choice.

The relationship between the environment and a species has an enormous impact on evolution. Structural and behavioural adaptations that lead to advantages, such as better feeding or locomotion strategies, increase reproductive success and allow these traits to persist and propagate. The evolution of the primate prehensile tail is an example of an environmental effect: the arboreal habitat guided a pre-existing trait to take on alternative functions.

The prehensile tail is a tail that can grasp with proficiency and suspend an animal's full body weight unassisted ${ }^{1}$. This trait has evolved a minimum of 14 distinct times in mammals ${ }^{2}$. In arboreal habitats, prehensility is advantageous to navigating and foraging between tree branches. The distribution of the prehensile tail in these habitats is evidence of an effective adaptation to life in the forest. Prehensile tails in primates are found exclusively in New World monkeys (Platyrrhini) that inhabit the dense, tropical forests of Central and South America ${ }^{3}$. The only primate genera (Figure 1) that possess prehensile tails are spider monkeys (Ateles), woolly spider monkeys (Brachyteles), howler monkeys (Alouatta), woolly monkeys (Lagorix), and capuchin monkeys

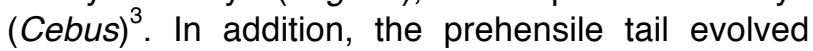
separately in the Atelidae family and the whiteheaded capuchin (C. capucinus) of the Cebidae family. Both groups share ancestry; however, there are morphological differences that suggest they arose from separate evolutionary events by means of parallel evolutionary paths.

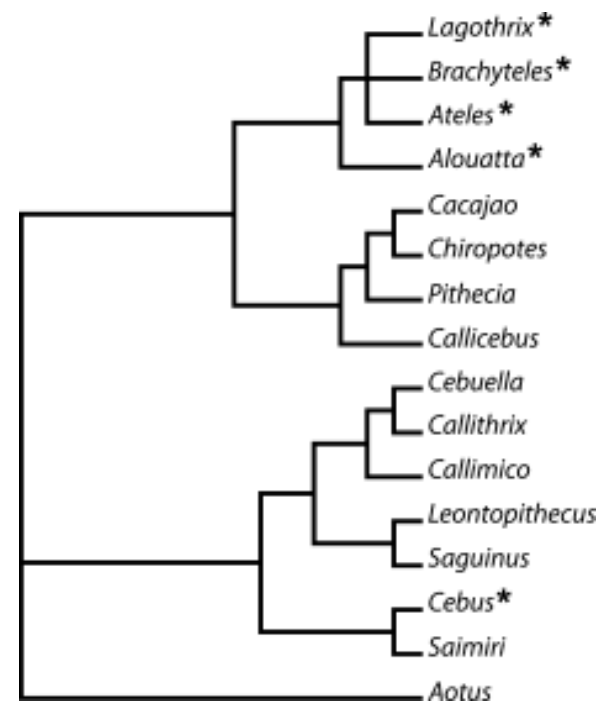

Figure 1 - A diagram showing phylogenetic relationships among the platyrrhine. Genera with prehensile tails are marked by an asterisk $\left({ }^{*}\right)^{3}$.

A proposed evolutionary path for the primate prehensile tail suggests that tail-assisted hind limb suspension was a transitional behaviour and an important stage in its evolution ${ }^{4}$. New World monkeys frequently use their hind limbs to suspend themselves while descending trees headfirst or 
hanging from branches. While hanging from their hind limbs, non-prehensile New World monkeys will forcefully brace their tail against a support, using their tail for more than just balance. Prehensile tailed New World monkeys took this behaviour a step further, and they can now suspend their weight using their tail only. In order to handle the mechanical loading force of supporting an animal's entire body mass, structural changes to the tail had to occur.

Numerous differences are present in the bone morphology between prehensile and nonprehensile primates, and these differences lead to superior suspension abilities in the prehensile primates. The primate tail can be divided into the proximal region and the distal region, which are two distinct sections with different structured vertebrae and joints. The proximal region is near the base of the tail, while the distal region spans the remaining length of the tail. Prehensile tails have vertebrae that are more robust with stronger bending and torsional strengths, where this difference is more evident in the distal region (Figure 2$)^{3}$. Prehensile primates use the distal region of their tail to suspend from branches, thus strong vertebrae are needed to support this force. The distal vertebrae of prehensile tails are also shorter in length than the distal vertebrae of nonprehensile tails, where the shortest vertebrae can be found two-thirds down the extent of the tail. The shortest vertebrae are located at the flexion point seen in a tail during hind limb suspension as it wraps itself against the support, indicating that this posture was a transitional behaviour leading to the evolution of the prehensile tail ${ }^{4}$.

A

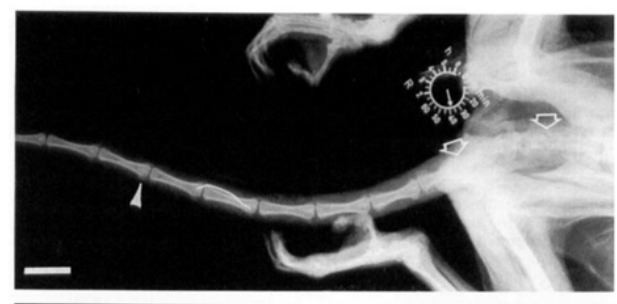

B

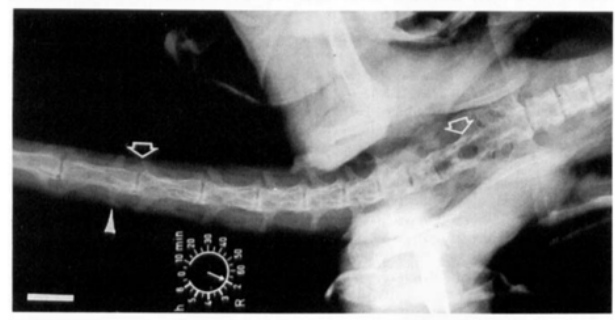

Figure 2 - X-ray comparison of tail vertebrae of $(A)$ a non-prehensile tailed primate (Macaca fasicularis) compared with (B) a prehensile tailed primate (Ateles paniscus) ${ }^{3}$.

The proximal region of the tail contains specialized zygapophyseal joints that have limited flexibility compared with the distal region's intervertebral disc articulation. A prehensile tail has a longer proximal region than a non-prehensile tail, thereby consisting of a greater number of proximal vertebrae as well as zygapophyseal joints ${ }^{2}$. Increased length leads to greater flexibility of the proximal region in prehensile tailed primates, while more zygapophyseal joints in series maintain rigidity during movement. Joints found in the tails of prehensile primates also have larger articular surfaces compared with the tails of non-prehensile primates. When the joint is in use, this larger articular surface area dissipates the applied forces, thereby reducing potential damage to the joint ${ }^{5}$. Primate prehensile tails also have more pronounced and convex articular surface curvatures, which are associated with an increased range of motion of the tail $^{5}$. Changes to the articular surface of a prehensile tail confer a greater capacity for loading and movement, which allows suspension using just the tail.

Muscle differences are evident as well between prehensile tails and non-prehensile tails in primates. Muscle attachment sites are larger in prehensile tails, thus there is a greater muscle expansion and subsequent bending movement when a flexing force is applied ${ }^{5}$. This is significant for prehensility because the tail must support a greater loading force. An important feature unique to the Atelidae is a naked tactile pad, which is found near the tip of the tail. The pad is hairless and especially sensitive to touch, which improves gripping power substantially. The tail of the white-headed capuchin (Cebus) is covered entirely in fur. This difference shows how the prehensile tail evolved in parallel between these two groups because each atelid has this bare pad, indicating a shared origin independent from that of the white-headed capuchin. However, these two groups share several internal aspects regarding their tails, showing how their arboreal habitats selected for specific structural features that led to the rise of the prehensile tail.

Primates with prehensile tails can exploit new opportunities for feeding and more efficient methods of locomotion, leading to selection for this trait. The prehensile tail illustrates how the environment can shape pre-existing structures to be used for new, advantageous behaviours. The 
morphological differences in the bone and muscle structure of the prehensile tail underlie the tail's functionality.

\section{References}

1. Fleagle JG. Primate Adaption and Evolution. 2nd ed. Cambridge (MA): Academic Press; 1998. p. 172.

2. Organ JM. Structure and function of platyrrhine caudal vertebrae. Anat Rec. 2010; 293(4):730-745. DOI: 10.1002/ar.21129

3. Lemelin P. Comparative and functional myology of the prehensile tail in New World monkeys. J Morphol. 1995; 224(3):351-368. DOI: 10.1002/jmor.1052240308

4. Meldrum DJ. Tail-assisted hind limb suspension as a transitional behavior in the evolution of the platyrrhine prehensile tail. In: Strasser E, Fleagle J, Rosenberger AL, McHenry $\mathrm{H}$, editors. Primate locomotion: recent advances. New York: Plenum Press; 1998. p. 145-156.

5. Deane AS, Russo GA, Muchlinski MN, Organ JM. Caudal vertebral body articular surface morphology correlates with functional tail use in anthropoid primates. J Morphol. 2014; 275(11):1300-1311. DOI: 10.1002/jmor.20304 